\title{
Quark-Meson Coupling Model for a Nucleon
}

\author{
S. W. Hong ${ }^{a}$, B. K. Jennings ${ }^{b}$ \\ ${ }^{a}$ Department of Physics and Institute of Basic Science, Sungkyunkwan University, \\ Suwon 440-746, Korea \\ ${ }^{b}$ TRIUMF, 4004 Wesbrook Mall, Vancouver, British Columbia, Canada V6T 2A3
}

\begin{abstract}
The quark-meson coupling model for a nucleon is considered. The model describes a nucleon as an MIT bag, in which quarks are coupled to scalar and vector mesons. A set of coupled equations for the quark and the meson fields are obtained and are solved in a self-consistent way. It is shown that the mass of a nucleon as a dressed MIT bag interacting with $\sigma$ - and $\omega$-meson fields significantly differs from the mass of a free MIT bag. A few sets of model parameters are obtained so that the mass of a dressed MIT bag becomes the nucleon mass. The results of our calculations imply that the self-energy of the bag in the quark-meson coupling model is significant and needs to be considered in doing the nuclear matter calculations.
\end{abstract}

PACS number: 24.85.+p, 21.65.+f, 12.39.Ba

\section{Introduction}

Quantum hadrodynamics [1] proved to be a very successful model in describing the bulk properties of nuclear matter as well as the properties of finite nuclei. The model is rather simple with only a few parameters, and yet it has been successfully applied to a vast number of nuclear matter and nuclear structure problems. On the other hand, this model has a fundamental shortcoming that the nucleon with a composite structure is treated as a point particle. Several years ago a model to remedy this problem was proposed by Guichon [2]. He proposed a quark-meson coupling (QMC) model and investigated the direct quark degrees of freedom in producing the nuclear saturation mechanism in nuclear matter. The model describes nuclear matter as non-overlapping MIT bags [3, 4] interacting through the self-consistent exchange of scalar and vector mesons in the meanfield approximation. In other words, the nucleon in nuclear matter is assumed to be a static spherical MIT bag in which quarks interact with the scalar $(\sigma)$ - and the vector $(\omega)$-meson fields. The model was refined later to include the nucleon Fermi motion and 
the center-of-mass corrections to the bag energy [5] and was applied to a number of problems $[6-11]$.

In the MIT bag model [3, 4] the bag constant $B$ and a phenomenological parameter $Z$ are fixed such that the nucleon mass of $939 \mathrm{MeV}$ is reproduced for some bag radius $R$. The bag constant $B$ produces the pressure to make a bubble in the QCD vacuum. $Z$ is to account for various corrections including the zero-point motion. In the QMC model the quark- $\sigma$ coupling constant $\left(g_{\sigma}^{q}\right)$ and the quark- $\omega$ coupling constant $\left(g_{\omega}^{q}\right)$ are introduced. Usually, $B$ and $Z$ are fixed first so that the mass of the free MIT bag becomes equivalent to the the nucleon mass of $939 \mathrm{MeV}$ for a certain bag radius. $B^{\text {free }}$ and $Z^{\text {free }}$ thus obtained are used in the QMC model calculations for nuclear matter problems. $g_{\sigma}^{q}$ and $g_{\omega}^{q}$ are then determined so as to reproduce the binding energy per nucleon (B.E. = $-16 \mathrm{MeV})$ at the saturation density $\left(\rho_{N}^{0}=0.17 \mathrm{fm}^{-3}\right)$.

However, due to the interaction between the quarks and the $\sigma$-and the $\omega$-mesons it is expected that the mass of a single dressed MIT bag in free space, when calculated with $B^{\text {free }}$ and $Z^{\text {free }}$ described above, would no longer be the nucleon mass of $939 \mathrm{MeV}$. Such a deviation of the mass of the dressed bag in free space has been neglected in previous QMC calculations in choosing the model parameters. If the deviation in mass is significant, it is necessary to modify the parameters to get the correct nucleon mass before implementing them in the nuclear matter calculations. It is this change in the mass of a dressed MIT bag that we address ourselves to in this paper. In Section 2 we describe the QMC model for a nucleon. A brief review of the QMC model for nuclear matter is given in Section 3 . Section 4 contains the results of the QMC model calculations for a nucleon and nuclear matter. The paper is summarized in Section 5.

\section{Quark-meson coupling model for a nucleon}

The Lagrangian density for the MIT bag in which quark fields $\psi$ are coupled to the $\sigma$ and $\omega$ - fields may be written as

$$
\begin{aligned}
\mathcal{L}= & {\left[\frac{i}{2}\left(\bar{\psi} \gamma^{\mu} \partial_{\mu} \psi-\left(\partial_{\mu} \bar{\psi}\right) \gamma^{\mu} \psi\right)-\bar{\psi}\left(g_{\omega}^{q} \gamma_{\mu} \omega^{\mu}+\left(m_{q}-g_{\sigma}^{q} \sigma\right)\right) \psi-B\right] \theta_{v} } \\
& -\frac{1}{2} \bar{\psi} \psi \Delta_{s}-\frac{1}{4} F_{\mu \nu} F^{\mu \nu}+\frac{1}{2} m_{\omega}^{2} \omega_{\mu} \omega^{\mu}+\frac{1}{2}\left[\left(\partial_{\mu} \sigma\right)\left(\partial^{\mu} \sigma\right)-m_{\sigma}^{2} \sigma^{2}\right],
\end{aligned}
$$

where $F_{\mu \nu}=\partial_{\mu} \omega_{\nu}-\partial_{\nu} \omega_{\mu}, \theta_{v}$ is the step function for confining the quarks inside the bag, and $\Delta_{s}$ is the $\delta$-function at the bag surface. We will neglect the isospin breaking and

take $m_{q}=\left(m_{u}+m_{d}\right) / 2$ hereafter. For numerical calculations $m_{q}$ will be taken to be zero. The mass of $\sigma(\omega)$ is taken as 550 (783) MeV. From this Lagrangian density the equations of motion for quark field $\psi$, sigma field $\sigma$, and omega field $\omega_{\mu}$ follow. For $\psi$ we have

$$
\left[\gamma^{\mu}\left(i \partial_{\mu}-g_{\omega}^{q} \omega_{\mu}\right)-\left(m_{q}-g_{\sigma}^{q} \sigma\right)\right] \psi \theta_{v}=\frac{1}{2}\left(1-i \gamma^{\mu} n_{\mu}\right) \psi \Delta_{s}
$$

The left hand side of this equation gives us the equation for the quarks inside the bag, and the right hand side provides us with the linear boundary condition at the bag surface 
$(r=R)$. The equations for $\sigma$ and $\omega_{\mu}$ are, respectively,

$$
\partial_{\mu} \partial^{\mu} \sigma+m_{\sigma}^{2} \sigma=g_{\sigma}^{q} \bar{\psi} \psi \theta_{v}
$$

and

$$
\partial^{\nu} F_{\nu \mu}+m_{\omega}^{2} \omega_{\mu}=g_{\omega}^{q} \bar{\psi} \gamma_{\mu} \psi \theta_{v}
$$

If we consider only the ground state quarks in the static spherical MIT bag and keep only the time-component of $\omega_{\mu}$, Eq. (2) becomes the following coupled linear differential equations for $g(r)$ and $f(r)$ inside the bag;

$$
\begin{aligned}
& \frac{d f(r)}{d r}=-\left[2 \frac{f(r)}{r}+E^{-}(r) g(r)\right] \\
& \frac{d g(r)}{d r}=E^{+}(r) f(r),
\end{aligned}
$$

where $g(r)$ and $f(r)$ are the radial parts of the upper and the lower components of $\psi$, i.e.,

$$
\psi(t, \mathbf{r})=e^{-i \epsilon_{q}^{0} t / R}\left(\begin{array}{c}
g(r) \\
-i \vec{\sigma} \cdot \hat{\mathbf{r}} f(r)
\end{array}\right) \frac{\chi_{q}}{\sqrt{4 \pi}},
$$

where $\vec{\sigma}$ is the Pauli spin matrix and $\chi_{q}$ is the quark spinor. Also,

$$
\begin{aligned}
& E^{+}(r)=\frac{\epsilon_{q}^{0}}{R}-g_{\omega}^{q} \omega_{0}(\mathbf{r})+\left(m_{q}-g_{\sigma}^{q} \sigma(\mathbf{r})\right) \\
& E^{-}(r)=\frac{\epsilon_{q}^{0}}{R}-g_{\omega}^{q} \omega_{0}(\mathbf{r})-\left(m_{q}-g_{\sigma}^{q} \sigma(\mathbf{r})\right)
\end{aligned}
$$

with $\sigma(\mathbf{r})$ and $\omega_{0}(\mathbf{r})$ being the $\sigma$-and the time component of the $\omega$-fields. Since we are dealing with the ground state, Eqs. (8) are the equations in the radial coordinate $r$ only. The linear boundary condition from Eq. (2), when rewritten by using Eq. (7), reads

$$
f\left(\frac{x_{q} r}{R}\right)=-g\left(\frac{x_{q} r}{R}\right)
$$

at the bag surface, $r=R$, and determines the eigenvalue $x_{q}$ of the quarks. $\epsilon_{q}^{0}$ is then given by $\sqrt{x_{q}^{2}+\left(R m_{q}\right)^{2}}=x_{q}$ for $m_{q}=0$.

In the static spherical approximation Eqs. (3) and (4) are reduced to

$$
\left(\nabla^{2}-m_{\sigma}^{2}\right) \sigma(\mathbf{r})=-g_{\sigma}^{q}\left(3 \rho_{s}\right) \theta(R-r)
$$

and

$$
\left(\nabla^{2}-m_{\omega}^{2}\right) \omega_{0}(\mathbf{r})=-g_{\omega}^{q}\left(3 \rho_{B}\right) \theta(R-r),
$$

respectively, where $\rho_{s}(=\bar{\psi} \psi)$ and $\rho_{B}\left(=\bar{\psi} \gamma_{0} \psi\right)$ are scalar and baryon densities, respectively, and 3 is multiplied by them to account for the sum over 3 quarks. Equations (5), (6), (10), and (11) constitute a set of coupled equations for $\psi, \sigma$, and $\omega_{0}$, which need to be solved self-consistently. 
By solving these equations we can obtain the eigenvalue of the quarks and the energy $\left(E_{N}\right)$ of the nucleon bag. $E_{N}$ can be computed by using

$$
E_{N}=\int d^{3} r T_{00}
$$

with

$$
T_{00}=\left(\frac{\mathcal{E}_{q}}{R} \bar{\psi} \gamma^{0} \psi+B\right) \theta(R-r)-\frac{1}{2}\left(\left(\nabla \omega_{0}\right)^{2}+\left(m_{\omega} \omega_{0}\right)^{2}\right)+\frac{1}{2}\left((\nabla \sigma)^{2}+\left(m_{\sigma} \sigma\right)^{2}\right),
$$

where $\mathcal{E}_{q}$ is given by

$$
\frac{\mathcal{E}_{q}}{R}=3 \frac{\epsilon_{q}^{0}}{R}-\frac{Z}{R}=3 \frac{x_{q}}{R}-\frac{Z}{R}
$$

with the sum over 3 quarks taken into account. Correcting for spurious center-of-mass motion in the bag, the mass of the nucleon bag at rest is taken to be [5, 6]

$$
M_{N}=\sqrt{E_{N}^{2}-\left\langle p_{c . m .}^{2}\right\rangle}
$$

where $\left\langle p_{c . m .}^{2}\right\rangle=\sum_{k=1}^{3}\left\langle p_{k}^{2}\right\rangle=3\left(x_{q} / R\right)^{2}$. By minimizing $M_{N}$ with respect to the bag radius $R$, we can get the nucleon mass and the bag radius.

\section{Quark-meson coupling model for nuclear matter}

Now, let us consider the QMC model for nuclear matter. Detailed formulations and justification of the model can be found in Refs. [2, 5, 6, 7], and here we only briefly sketch the model for later discussions. In addition to the static spherical approximation assumed in Section 2 we now further use the mean-field approximation. From Eq. (2) the quark field $\psi$ inside the bag in the mean-field approximation may be written as

$$
\left[i \gamma^{\mu} \partial_{\mu}-g_{\omega}^{q} \bar{\omega} \gamma^{0}-\left(m_{q}-g_{\sigma}^{q} \bar{\sigma}\right)\right] \psi=0
$$

where $\bar{\sigma}$ and $\bar{\omega}$ are the mean-fields for $\sigma$-and $\omega$-mesons, respectively. Eq. (16) has the following simple solution,

$$
\psi(t, \mathbf{r})=\mathcal{N} e^{-i \epsilon_{q} t / R}\left(\begin{array}{c}
j_{0}(x r / R) \\
i \beta_{q} \vec{\sigma} \cdot \hat{\mathbf{r}} j_{1}(x r / R)
\end{array}\right) \frac{\chi_{q}}{\sqrt{4 \pi}},
$$

where $\epsilon_{q}=\Omega_{q}+g_{\omega}^{q} \bar{\omega} R$,

$$
\beta_{q}=\sqrt{\frac{\Omega_{q}-R m_{q}^{*}}{\Omega_{q}+R m_{q}^{*}}}, \quad \mathcal{N}^{-2}=2 R^{3} j_{0}^{2}(x)\left[\Omega_{q}\left(\Omega_{q}-1\right)+R m_{q}^{*} / 2\right] / x^{2}
$$

with $\Omega_{q}=\sqrt{x^{2}+\left(R m_{q}^{*}\right)^{2}}$ and $m_{q}^{*}=m_{q}-g_{\sigma}^{q} \bar{\sigma}$. The boundary condition

$$
j_{0}(x r / R)=\beta_{q} j_{1}(x r / R)
$$


at $r=R$ gives us the $x$ value. Then the energy of the bag can be expressed as

$$
E_{b a g}=3 \frac{\Omega_{q}}{R}-\frac{Z}{R}+\frac{4}{3} \pi R^{3} B
$$

and the effective mass of the bag in nuclear matter is taken as

$$
M_{b a g}^{*}=\sqrt{E_{b a g}^{2}-\left\langle p_{c . m .}^{2}\right\rangle} .
$$

Using the minimum condition

$$
\frac{\partial M_{b a g}^{*}}{\partial R}=0
$$

we can determine the effective mass.

The total energy per nucleon at nuclear matter density, $\rho_{N}$, including the Fermi motion of the nucleons, can be written as [5, 6]

$$
E_{t o t}=\frac{\gamma}{(2 \pi)^{3} \rho_{N}} \int^{k_{F}} d^{3} k \sqrt{M_{b a g}^{* 2}+\mathbf{k}^{2}}+\frac{\left(3 g_{\omega}^{q}\right)^{2}}{2 m_{\omega}^{2}} \rho_{N}+\frac{m_{\sigma}^{2}}{2 \rho_{N}} \bar{\sigma}^{2},
$$

where the spin-isospin degeneracy $\gamma$ is 4 for symmetric nuclear matter.

The mean field $\bar{\omega}$ created by the nucleons is given by [1, 2, 5, 6]

$$
\bar{\omega}=\frac{3 g_{\omega}^{q} \rho_{N}}{m_{\omega}^{2}}
$$

and the scalar mean field $\bar{\sigma}$ is determined by the thermodynamic condition

$$
\left(\frac{\partial E_{t o t}}{\partial \bar{\sigma}}\right)_{R, \rho_{N}}=0
$$

which yields the self-consistency equation

$$
\bar{\sigma}=\frac{3 g_{\sigma}^{q}}{m_{\sigma}^{2}} C(\bar{\sigma}) \frac{\gamma}{(2 \pi)^{3}} \int^{k_{F}} d^{3} k \frac{M_{b a g}^{*}}{\sqrt{M_{b a g}^{* 2}+\mathbf{k}^{2}}}
$$

with

$$
3 g_{\sigma}^{q} C(\bar{\sigma})=3 g_{\sigma}^{q} \frac{E_{b a g}}{M_{b a g}^{*}}\left[\left(1-\frac{\Omega_{q}}{E_{b a g} R}\right) S(\bar{\sigma})+\frac{m_{q}^{*}}{E_{b a g}}\right]
$$

and

$$
S(\bar{\sigma})=\frac{\Omega_{q} / 2+R m_{q}^{*}\left(\Omega_{q}-1\right)}{\Omega_{q}\left(\Omega_{q}-1\right)+R m_{q}^{*} / 2} .
$$

Equation (26) is also to be solved consistently with Eq. (19). 


\section{Results and Discussions}

We first present the results for a single nucleon. In presenting the results we take the cases in which the bag radius is $1 \mathrm{fm}$. In Fig. 1 the nucleon mass $M_{N}$ is plotted as a function of the bag radius $R$. The solid curve represents the free MIT bag mass $\left(M_{N}^{\text {free }}\right)$ without a coupling of the quarks with the mesons; $g_{\sigma}^{q}=g_{\omega}^{q}=0$. In the calculations $B^{1 / 4}$ and $Z$ are taken to be $136.3 \mathrm{MeV}$ and 1.153 [6], respectively, which give us the minimum of $M_{N}^{\text {free }}$ as $939 \mathrm{MeV}$ at $1.0 \mathrm{fm}$. If we include the interaction of quarks with meson fields using the coupling constants $g_{\sigma}^{q}=5.605$ and $g_{\omega}^{q}=1.152$ chosen by Saito and Thomas[G], for instance, a solution to the field equations (5), (6), (10), and (11) does not exist. In Eq. (6) both $d g(r) / d r$ and $f(r)$ are negative (or zero) for all radii, and thus $E^{+}(r)$ needs to be positive. However, due to the large attraction coming from the large $g_{\sigma}^{q}$ value in relative to the small $g_{\omega}^{q}$ the eigenvalue $\epsilon_{q}^{0}$ in Eq. (8) becomes relatively small, which results in $E^{+}(r)$ being negative for this set of parameters; hence the non-existence of the solution. Therefore we show here results for other sets of parameters.

First, we include only the coupling of the quarks with the $\sigma$-meson; $g_{\omega}^{q}=0$. The bag mass decreases due to the attractive $\sigma$-coupling as shown in Fig. 1 by the thin dashed and the thin dash-dotted curves, respectively, for $g_{\sigma}^{q}=2$ and $4 . M_{N}$ is reduced to 930 and $903 \mathrm{MeV}$, and the bag radius also slightly decreases to 0.997 and $0.979 \mathrm{fm}$, respectively, for $g_{\sigma}^{q}=2$ and 4 . On the other hand, when the quarks are allowed to couple only with the $\omega$-field, i.e., $g_{\sigma}^{q}=0, M_{N}$ increases as plotted by the thick dashed and the thick dash-dotted curves, respectively, for $g_{\omega}^{q}=2$ and $4 . M_{N}$ is pushed up to 954 and $994 \mathrm{MeV}$ due to the repulsive coupling, and the bag radius becomes 1.00 and $1.03 \mathrm{fm}$, respectively.

If the quarks are allowed to couple with both the $\sigma$-and the $\omega$-mesons, cancellation between the attraction and the repulsion takes place. In Fig. 2 we show $M_{N}$ calculated with $g_{\sigma}^{q}=g_{\omega}^{q}=2$ by the dashed curve in comparison with $M_{N}^{\text {free }}\left(g_{\sigma}^{q}=g_{\omega}^{q}=0\right)$ plotted by the solid curve. $M_{N}$ for $g_{\sigma}^{q}=g_{\omega}^{q}=2$ has a minimum at $946 \mathrm{MeV}$ and $R=1.01 \mathrm{fm}$. The mass difference $(946-939=7 \mathrm{MeV})$ is just about what is left after the cancellation between the thin dashed curve $\left(g_{\sigma}^{q}=2, g_{\omega}^{q}=0\right)$ and the thick dashed curve $\left(g_{\sigma}^{q}=0\right.$, $\left.g_{\omega}^{q}=2\right)$ in Fig. 1. $M_{N}$ calculated with $g_{\sigma}^{q}=g_{\omega}^{q}=4$ is also plotted in Fig. 2 by the dash-dotted curve. The minimum of $M_{N}$ is $964 \mathrm{MeV}$ at $R=1.02 \mathrm{fm}$. Again, the mass shift $(964-939=15 \mathrm{MeV})$ is just about the repulsion left after the cancellation between the two dash-dotted curves in Fig. 1. It is worth noting here that when we use the same value for $g_{\sigma}^{q}$ and $g_{\omega}^{q}$ the net result is a repulsion. This may be understood by looking at the meson fields. In Fig. $3 \sigma(\mathbf{r})$ and $\omega_{0}(\mathbf{r})$ are plotted as a function of radius for a fixed bag radius $R=1 \mathrm{fm}$ when $g_{\sigma}^{q}=g_{\omega}^{q}=2$. The figure shows that the shapes of $\sigma(\mathbf{r})$ and $\omega_{0}(\mathbf{r})$ are largely dictated by those of the source densities $\rho_{s}$ and $\rho_{B}$, respectively. Just as $\rho_{B}$ is bigger than $\rho_{s}$ at larger radii, $\omega_{0}(\mathbf{r})$ is greater than $\sigma(\mathbf{r})$ at larger radii, which is the significant radial region. Thus, when the two coupling constants are equal, a net effect turns out to be repulsive.

In the nuclear matter at normal nuclear densities the internucleon distance is about $2 \mathrm{fm}$. In the mean-field approximation meson fields are assumed to be constant in the 
matter. On the other hand, Fig. 3 (a) shows that the range of the meson fields is roughly around $1.5 \mathrm{fm}$ for both mesons. The fields become almost negligible at $r \approx 2$ $\mathrm{fm}$. However, since the nucleons in nuclear matter are in the Fermi motion, the meson fields with the range of about $1.5 \mathrm{fm}$ may be approximated to mean-fields in the matter. Therefore, the present study of a single nucleon in the QMC model seems compatible with the QMC model for the nuclear matter, which will be discussed shortly.

We then looked for parameters that could give us the correct nucleon mass for $R=1$ fm. First we fixed $B^{1 / 4}$ and $Z$ as $136.3 \mathrm{MeV}$ and 1.153 [6], respectively, and searched for $g_{\sigma}^{q}$ and $g_{\omega}^{q}$. It was not possible to find $g_{\sigma}^{q}$ and $g_{\omega}^{q}$ that would yield the correct nucleon mass with the given values of $B^{1 / 4}$ and $Z$. When we let $Z$ also vary, keeping $B^{1 / 4}$ fixed as $136.3 \mathrm{MeV}$, it was still impossible to find parameters producing the correct nucleon mass. On the other hand, when we let $B^{1 / 4}$ vary, keeping $Z$ as 1.153 , we could obtain the correct mass with the parameter set 2 listed in Table 1 . There are four parameters to be fixed, whereas there are only two quantities to fit; the nucleon mass and the bag radius. Thus the parameters are not unique. We list in Table 1 other sets of parameters that can produce the nucleon mass of $939 \mathrm{MeV}$ for $R=1 \mathrm{fm}$. $M_{N}$ 's calculated with the parameter sets $2-7$ are all indistinguishable from the solid curve in Fig. 1 or Fig. 2, so they are not plotted here. One can see from Table 1 that $g_{\sigma}^{q}$ is not much larger than $g_{\omega}^{q} \cdot g_{\sigma}^{q}$ is often only slightly bigger than $g_{\omega}^{q}$, as the relativistic Dirac phenomenolgy has shown. In fact, this is expected from Fig. 2, which shows that with the equal values of $g_{\sigma}^{q}$ and $g_{\omega}^{q}$ there is only a small repulsion. To restore the correct mass, $g_{\sigma}^{q}$ would have to be a little larger than $g_{\omega}^{q}$. This is in contrast to the values of $g_{\sigma}^{q}$ and $g_{\omega}^{q}$ previously obtained by QMC model calculations for nuclear matter. Too large $g_{\sigma}^{q}$ values compared to small $g_{\omega}^{q}$ were one of the concerns in the previous results of the QMC model calculations.

Similar studies were made at other bag radii ranging from 0.6 to $1.0 \mathrm{fm}$, and similar results were obtained. Our results show that the change in the nucleon mass due to the coupling of the quarks with the mesons is not negligible. Such self-energy effects on the nucleon mass need to be taken into consideration in choosing the parameters for the calculation of nuclear matter properties. Thus, we performed the nuclear matter calculations as sketched in Section 3 along with the calculation for a nucleon. As mentioned earlier, when we use $g_{\sigma}^{q}$ and $g_{\omega}^{q}$ taken from Ref. [6], a solution for a nucleon problem does not exist. On the other hand, if we use the parameter sets 2 - 7 in Table 1, the calculated binding energies are not correct. Even saturation does not occur for these parameter sets. Therefore, we attempted to look for a new set of parameters that could reproduce simultaneously the nucleon mass and the nuclear binding energy. We found that it was not possible to get such parameters, at least, within the present scheme. Therefore, this additional requirement of reproducing a single nucleon mass can be used as a guide in refining the QMC model for nuclear matter. 


\section{Summary}

Recently the QMC model has been frequently used to describe the properties of nuclear matter and finite nuclei. However, in the previous calculations the change in the mass of the bag due to the self-energy has been ignored. Thus we have applied the QMC model to a single nucleon. Our calculations suggest that this change in the nucleon mass is not negligible. Therefore, we performed the QMC model calculations not only for a nucleon but also for nuclear matter and looked for parameters that would give us both the nucleon mass and the nuclear binding energy correctly. However, we only found that it was not possible to obtain such parameters within the present framework of the model. Taking into account this change in the nucleon mass may provide us with an additional constraint on the model.

\section{Acknowledgements}

This work was supported by the Natural Sciences and Engineering Research Council of Canada. SWH acknowledges kind hospitality at TRIUMF. He also thanks Prof. T. Udagawa for helpful discussions and was partially supported by Sungkyun Faculty Research Fund and BK21 Physics Research Division.

\section{References}

[1] B. D. Serot and J. D. Walecka, Adv. Nucl. Phys. 16 (1986) 1.

[2] P. A. M. Guichon, Phys. Lett. B 200 (1988) 235.

[3] A. Chodos, R. L. Jaffe, K. Johnson, C. B. Thorn, and V. Weisskopf, Phys. Rev. D 9 (1974) 3471; A. Chodos, R. L. Jaffe, K. Johnson and C. B. Thorn, Phys. Rev. D $10(1974) 2599$.

[4] A. W. Thomas, Adv. Nucl. Phys. 13 (1984) 1.

[5] S. Fleck, W. Bentz, K. Shimizu, and K. Yazaki, Nucl. Phys. A 510 (1990) 731.

[6] K. Saito and A. W. Thomas, Phys. Lett. B 327 (1994) 9.

[7] K. Saito and A. W. Thomas, Phys. Lett. B 335 (1994) 17; 363 (1995) 157; Phys. Rev. C 51 (1995) 2757; 52 (1995) 2789.

[8] A. W. Thomas, A. Michels, A. W. Schreiber, and P. A. M. Guichon, Phys. Lett. B 233 (1989) 43; K. Saito, A. Michels, and A. W. Thomas, Phys. Rev. C 46 (1992) R2149; K. Saito and A. W. Thomas, Nucl. Phys. A 574 (1994) 659.

[9] H. Q. Song and R. K. Su, Phys. Lett. B 358 (1995) 179. 
[10] X. Jin and B. K. Jennings, Phys. Lett. B 374 (1996) 13; Phys. Rev. C 54 (1996) 1427.

[11] H. Müller and B. K. Jennings, Nucl. Phys. A 640 (1998) 55. 
Table 1: The parameter set 1 is taken from Ref. [6]. The paremeter sets $2-7$ are the parameters that can produce $M_{N}=939 \mathrm{MeV}$ at the bag radius $R=1 \mathrm{fm}$. These parameters are not unique, so here we list some of them.

\begin{tabular}{c|ccccc}
\hline \hline set & $B^{1 / 4}(\mathrm{MeV})$ & $Z$ & $g_{\sigma}^{q}$ & $g_{\omega}^{q}$ & $M_{N}(\mathrm{MeV})$ \\
\hline 1 & 136.3 & 1.153 & 5.605 & 1.152 & No solution. \\
\hline 2 & 136.5 & 1.153 & 3.396 & 2.490 & 939 \\
\hline 3 & 136.5 & 1.169 & 3.300 & 2.616 & 939 \\
\hline 4 & 136.5 & 1.183 & 3.199 & 2.723 & 939 \\
\hline 5 & 136.5 & 1.199 & 2.941 & 2.760 & 939 \\
\hline 6 & 136.5 & 1.170 & 1.965 & 1.752 & 939 \\
\hline 7 & 136.4 & 1.238 & 3.840 & 3.746 & 939 \\
\hline
\end{tabular}


Figure 1: The nucleon mass $\left(M_{N}\right)$ calculated by the QMC model for a nucleon is plotted as a function of the bag radius $R$ for different coupling constants $g_{\sigma}^{q}$ and $g_{\omega}^{q} \cdot B^{1 / 4}=136.3$ $\mathrm{MeV}$ and $Z=1.153$ are used.

Figure 2: The nucleon mass $\left(M_{N}\right)$ calculated by the QMC model for a nucleon is plotted as a function of the bag radius $R$ for different coupling constants $g_{\sigma}^{q}$ and $g_{\omega}^{q} \cdot B^{1 / 4}=136.3$ $\mathrm{MeV}$ and $Z=1.153$ are used.

Figure 3: (a) The $\sigma$ - and the $\omega$-meson fields calculated by the QMC model for a nucleon are plotted as a function of radius $r$ for bag radius $R=1 \mathrm{fm}$ with $g_{\sigma}^{q}=g_{\omega}^{q}=2$, $B^{1 / 4}=136.3 \mathrm{MeV}$ and $Z=1.153$. (b) The scalar and the baryon densities are plotted by the dashed and the solid curves, respectively, with the same parameters. 


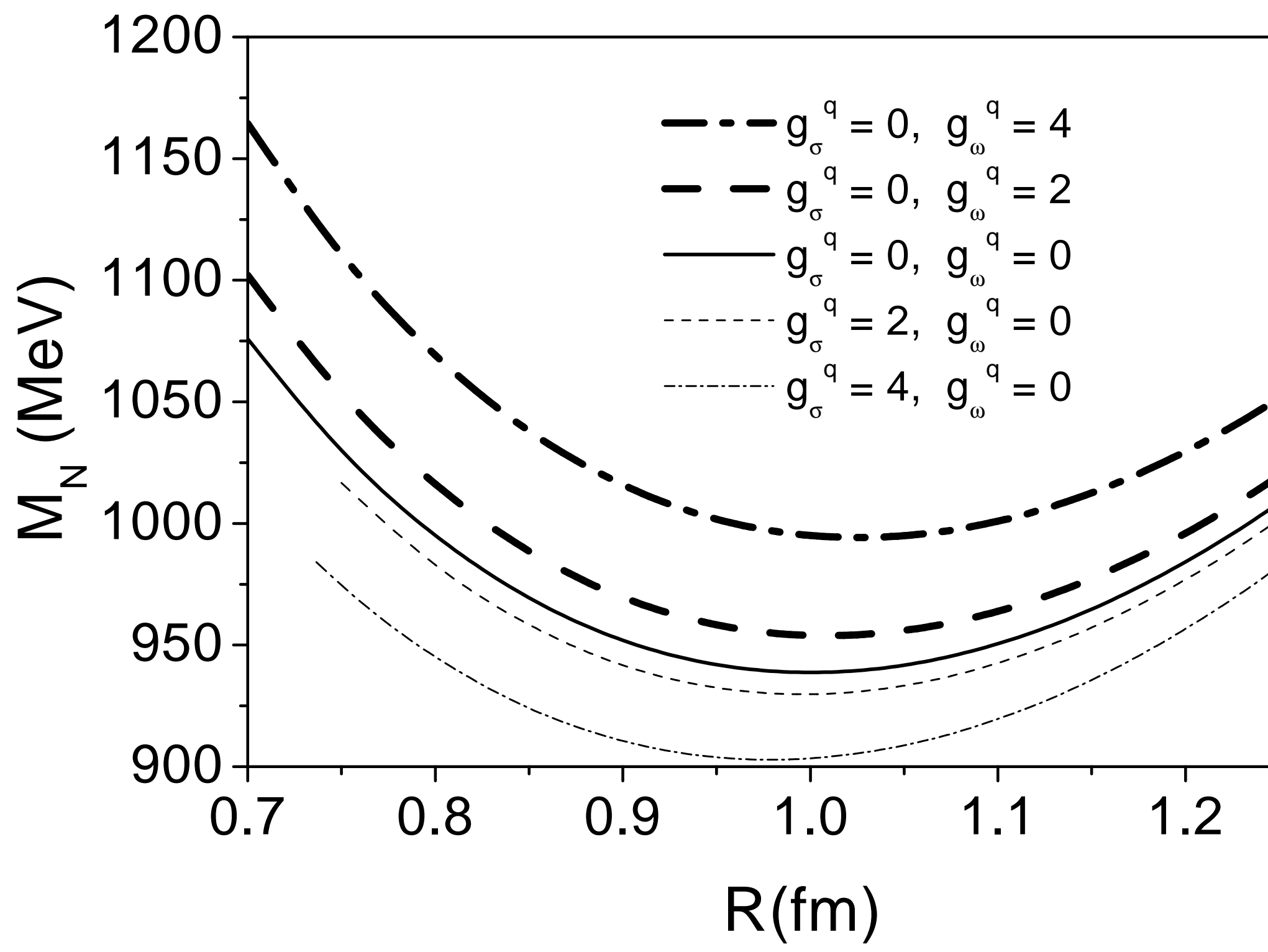

Fig. 


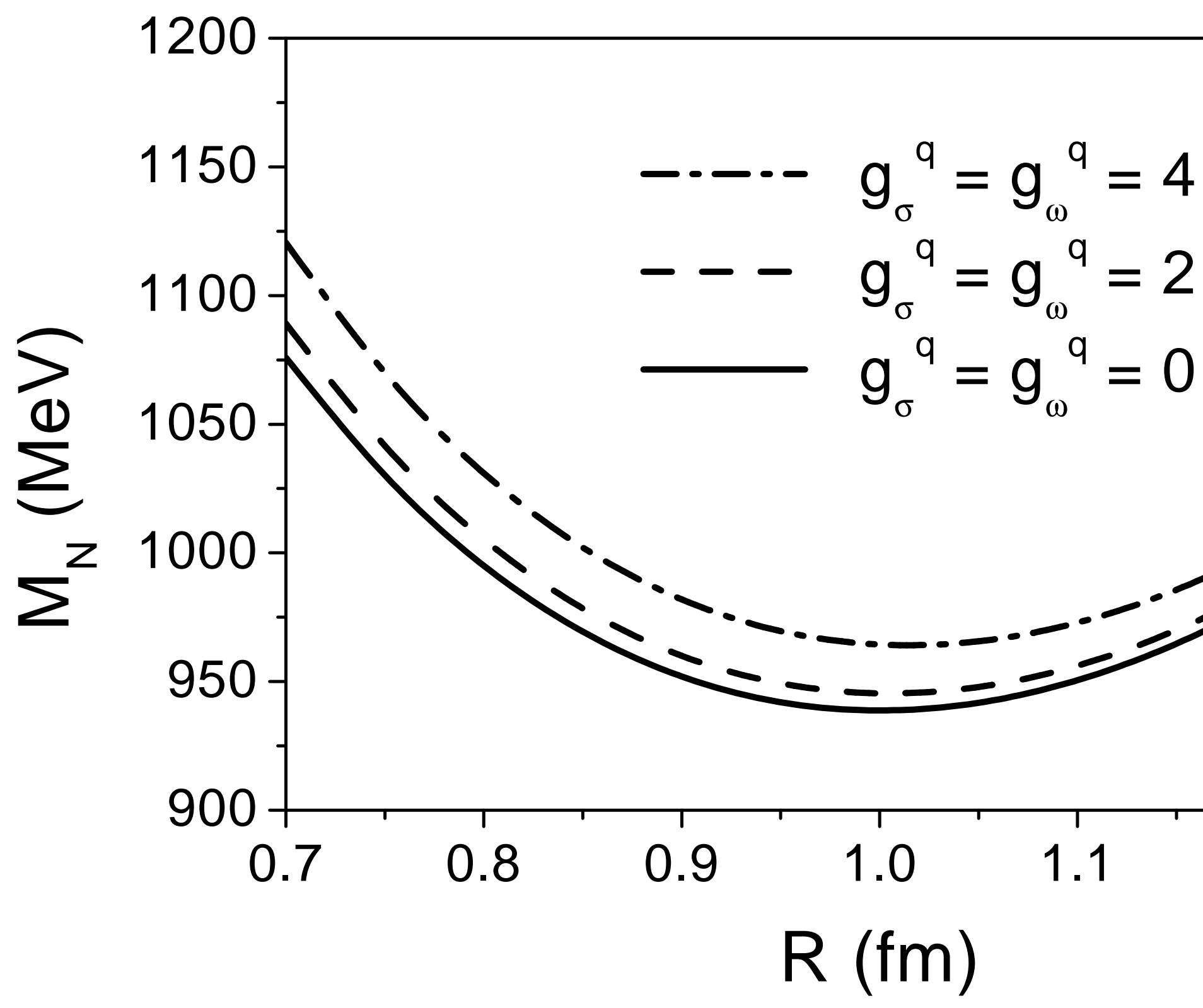




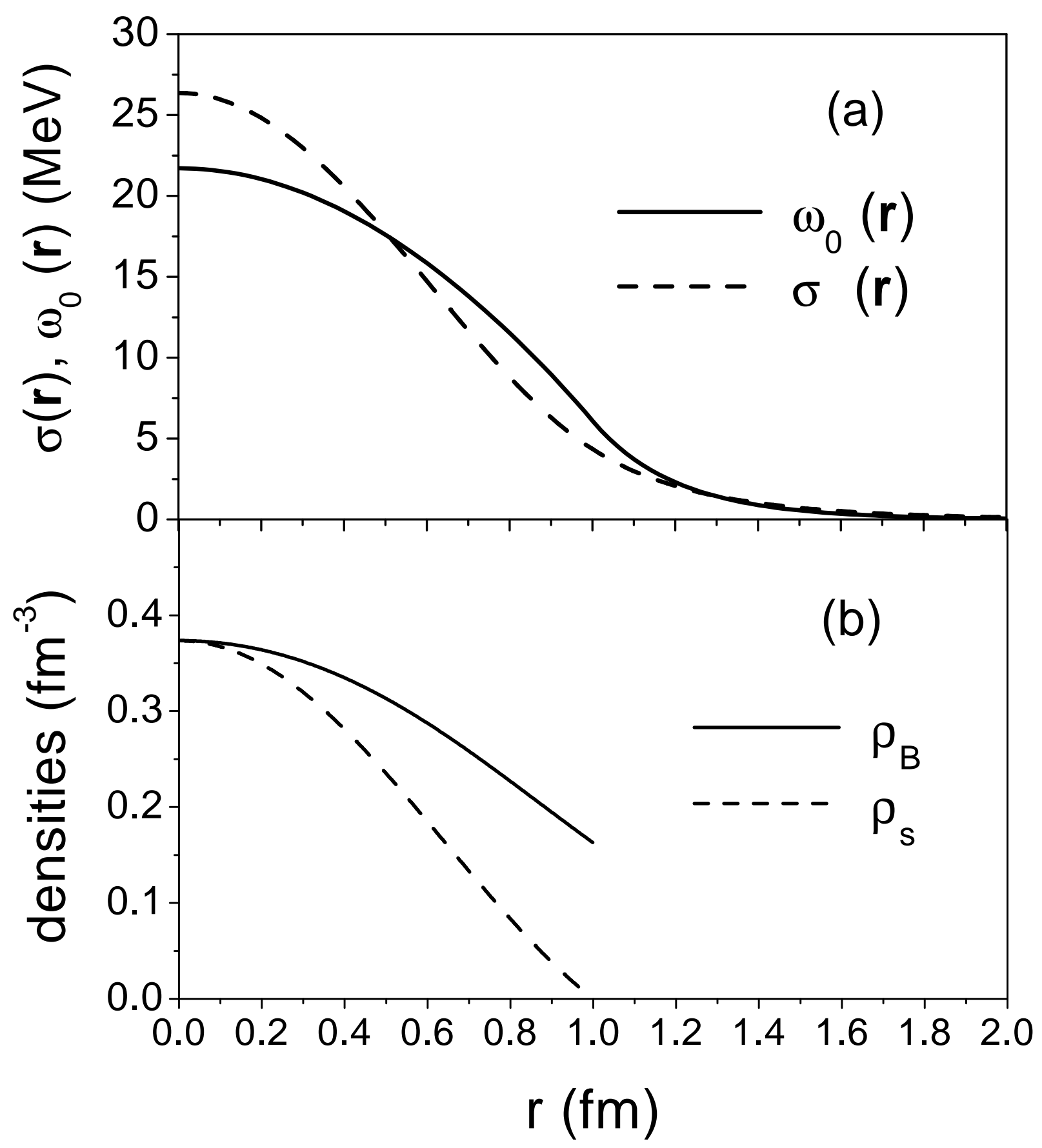

Fig. 3 